\title{
Measurement of Thermal Conductivity of PbTe Nanocrystal Coated Glass Fibers by the $3 \omega$ Method
}

Scott W. Finefrock, ${ }^{\dagger, \#}$ Yan Wang, ${ }^{\dagger, \#}$ John B. Ferguson, ${ }^{\S}$ James V. Ward, ${ }_{\dagger}^{\dagger}$ Haiyu Fang,

Jonathan E. Pfluger, ${ }^{\dagger}$ Douglas S. Dudis, ${ }^{\S}$ Xiulin Ruan, ${ }^{*}, \stackrel{\ddagger}{ }$ and Yue $\mathrm{Wu}^{*}{ }^{\dagger}$

${ }^{\dagger}$ School of Chemical Engineering, Purdue University, West Lafayette, Indiana 47907, United States

${ }^{\ddagger}$ School of Mechanical Engineering and the Birck Nanotechnology Center, Purdue University, West Lafayette, Indiana 47907, United States

${ }^{\S}$ Nanoelectronic Materials Branch, Materials and Manufacturing Directorate, Air Force Research Laboratory, Wright-Patterson Air Force Base, Dayton, Ohio 45433, United States

Supporting Information

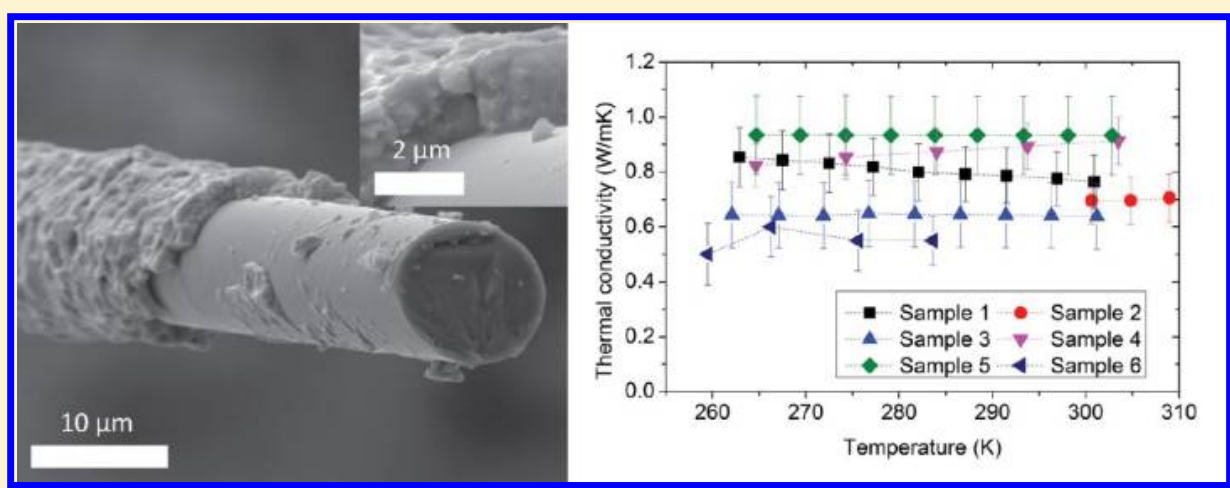

ABSTRACT: Fiber-based thermoelectric materials can conform to curved surfaces to form energy harvesting devices for waste heat recovery. Here we investigate the thermal conductivity in the axial direction of glass fibers coated with lead telluride ( $\mathrm{PbTe}$ ) nanocrystals using the self-heated $3 \omega$ method particularly at low frequency. While prior $3 \omega$ measurements on wire-like structures have only been demonstrated for high thermal conductivity materials, the present work demonstrates the suitability of the $3 \omega$ method for PbTe nanocrystal coated glass fibers where the low thermal conductivity and high aspect ratio result in a significant thermal radiation effect. We simulate the experiment using a finite-difference method that corrects the thermal radiation effect and extract the thermal conductivity of glass fibers coated by PbTe nanocrystals. The simulation method for radiation correction is shown to be generally much more accurate than analytical methods. We explore the effect of nanocrystal volume fraction on thermal conductivity and obtain results in the range of $0.50-0.93 \mathrm{~W} / \mathrm{mK}$ near room temperature.

KEYWORDS: Thermoelectric, flexible, thermal conductivity, $3 \omega$, radiation, finite difference method

$\mathrm{F}$ lexible thermoelectric materials such as nanocomposites ${ }^{1}$ and fibers ${ }^{2}$ can significantly impact waste heat recovery and solid-state cooling because of the advantages of lightweight, flexibility, and higher tolerance to thermal expansion. Particularly, flexibility can allow thermoelectric devices to be installed on curved surfaces such as automobile exhaust pipes, power plant steam pipes, manufacturing industry cooling pipes, and so forth. Our previous studies ${ }^{2}$ showed that thermoelectric fibers made from glass fibers coated with an ultrathin layer of $\mathrm{PbTe}$ nanocrystals (300 nm thick) can possess a similar thermoelectric figure of merit compared to traditional rigid bulk bismuth telluride modules. The module assemblies require several millimeters of thickness, which highlights a great potential for high performance with much reduced raw material cost for the coated fibers. In this paper, we present an investigation of the thermal conductivity of our thermoelectric fibers at the single fiber level using the $3 \omega$ method to further understand the thermal transport in the axial direction. Through simulations based on the finite-difference method, we successfully model the radiation effect and extract the thermal conductivity at the single fiber level. Our simulation method for radiation correction appears to be much more accurate than conventional analytical methods. Furthermore, we measure and simulate several samples to determine the thermal conductivities of composite fibers of varying composition; from $0 \% \mathrm{PbTe}$ to $35.8 \% \mathrm{PbTe}$ by volume fraction.

Several methods have been developed to measure the thermal properties of wire-like samples in the axial direction. These include the self-heating $3 \omega$ method, ${ }^{3} \mathrm{dc}$ thermal bridge

Received: February 12, 2013

Revised: October 15, 2013

Published: October 22, 2013 


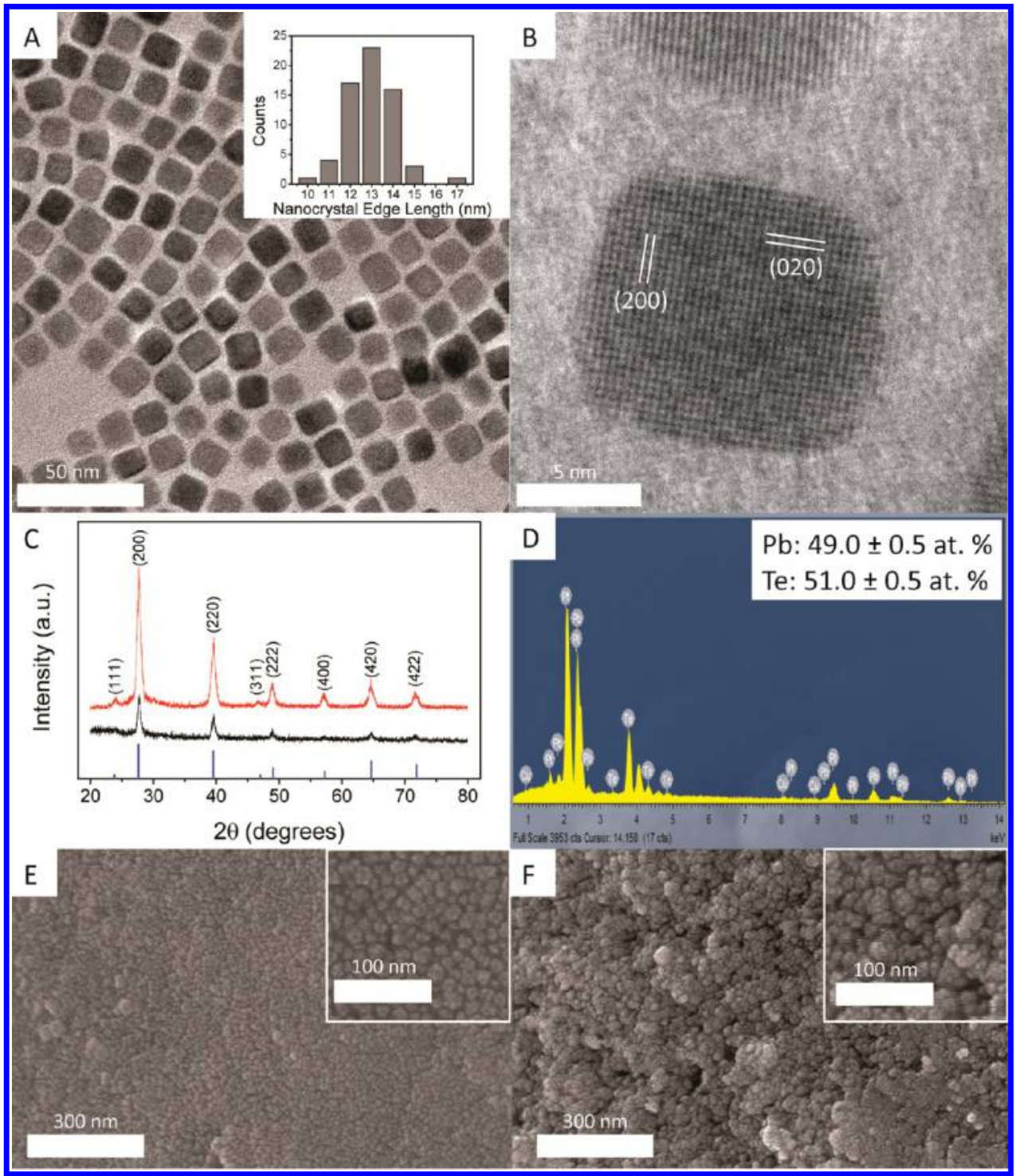

Figure 1. (A) Low magnification TEM image of PbTe nanocrystals with the inset of nanocrystal size distribution. (B) HRTEM image of a typical $\mathrm{PbTe}$ nanocrystal, showing the lattice fringes corresponding to the $\{200\}$ planes (JCPDS 38-1435). (C) XRD pattern of as-synthesized PbTe nanocrystals (upper curve) and annealed PbTe nanocrystal coated fibers (lower curve). (D) EDX spectrum of as-synthesized nanocrystals. The copper and platinum peaks are due to the substrate and conductive coating, respectively. (E) High-magnification SEM image of a coated fiber before annealing. (F) High magnification SEM image of a coated fiber after annealing.

method, ${ }^{4}$ thermal flash method, ${ }^{5}$ optical heating and electrical thermal sensing, ${ }^{6}$ pulsed laser-assisted thermal relaxation technique, ${ }^{7}$ and the technique of measuring a suspended wire over a microfabricated device. ${ }^{8-11}$ The $3 \omega$ method has experienced particularly widespread use on samples with high thermal conductivity, such as carbon nanotube bundles, ${ }^{3}$ carbon fibers ${ }^{12}$ and yarns, ${ }^{13}$ platinum wires, ${ }^{14}$ and polycrystalline silicon microbridges. ${ }^{15}$ However, the $3 \omega$ method has not yet been applied to low thermal conductivity wire-like materials such as PbTe-coated glass fibers. Furthermore, while the $3 \omega$ method employing a line heater on a substrate has been extended to layered composite planar samples, ${ }^{16-18}$ the selfheated wire $3 \omega$ technique has not yet been employed to determine the thermal properties of composite fibers. This work discusses the first $3 \omega$ experiments on composite fibers as well as important radiation effects that future work should consider when measuring the thermal properties of low thermal conductivity high aspect ratio fiber composites such as layered polymer fibers $^{19,20}$ and layered oxide fibers. ${ }^{21-23}$

The fabrication of the PbTe-coated thermoelectric glass fibers follows the procedure described in our previous studies. ${ }^{2}$ First, we synthesize $\mathrm{PbTe}$ nanocrystals by hot injection of trioctylphosphine-tellurium (TOP-Te) into a lead oleate solution. The chemicals including 1-octadecene (ODE, 90\%), oleic acid (OA, 90\%), lead(II) oxide (PbO, 99.9+\%), tellurium powder (99.8\%), hexane (98.5\%), acetone (99.5\%), chloroform (99\%), hydrazine hydrate solution (80\%), and acetonitrile (99.8\%) were purchased from Sigma-Aldrich and used without further purification. Tri- $n$-octylphosphine (TOP, 97\%) was purchased from Strem, Corning glass wool (fiber) was purchased from VWR, and Epo-tek H20E silver epoxy was purchased from Ted Pella.

A $0.37 \mathrm{M}$ TOP-Te solution is prepared in a nitrogen glovebox by dissolving $0.191 \mathrm{~g}$ of tellurium powder in $2 \mathrm{~mL}$ of TOP by heating at $57^{\circ} \mathrm{C}$ and then diluting with $2 \mathrm{~mL}$ of 


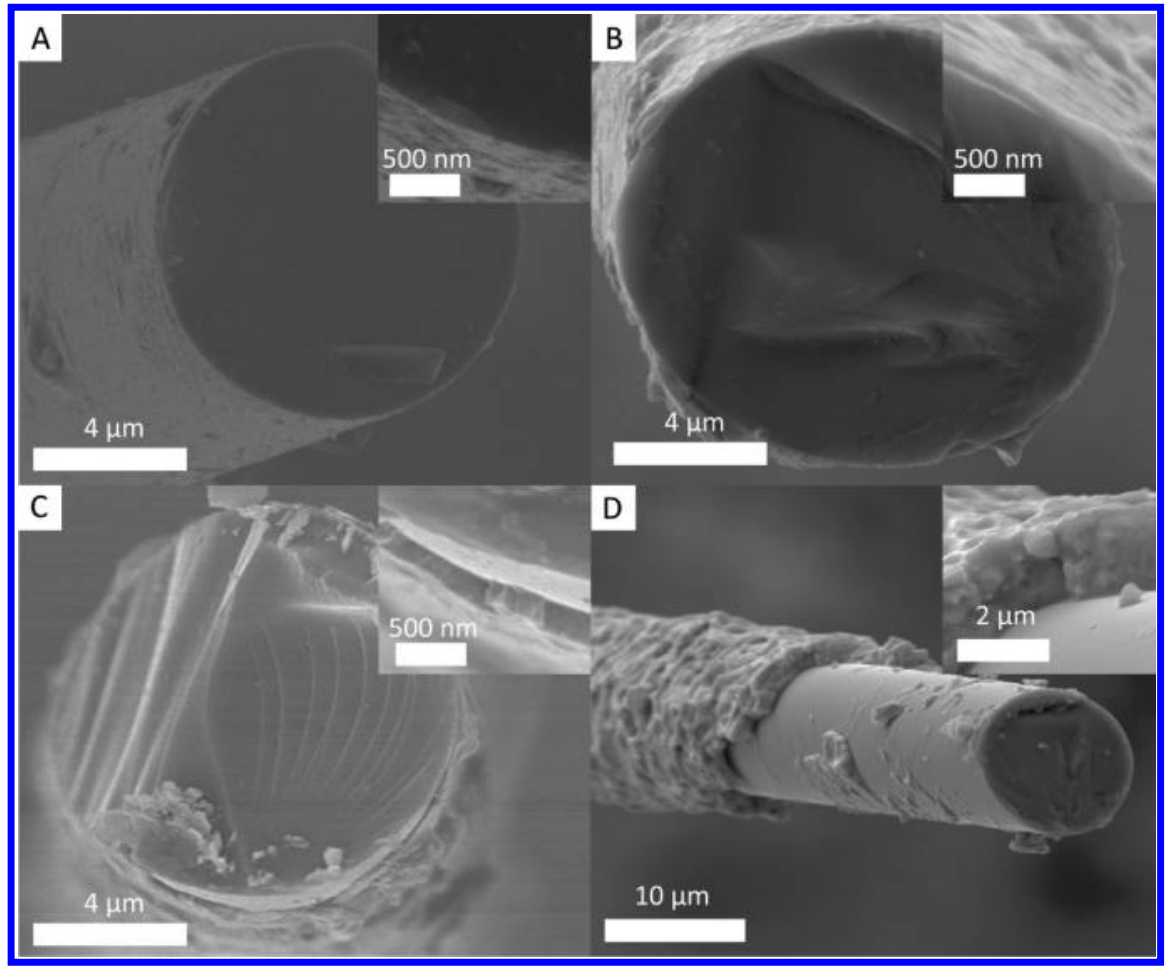

Figure 2. Cross section SEM images of cut ends of glass fibers. (A) Sample 1: 0\% PbTe. (B) Sample 4: 2.9\% PbTe. (C) Sample 5: 5.8\% PbTe. (D) Sample 6: $35.8 \%$ PbTe. Insets reveal the coating cross sections used for thickness measurements.

degassed ODE. The lead oleate solution is prepared by dissolving $0.223 \mathrm{~g}$ of $\mathrm{PbO}$ powder in $0.75 \mathrm{~mL}$ of $\mathrm{OA}$ and 12.7 $\mathrm{mL}$ of ODE in a $50 \mathrm{~mL}$ round-bottom flask and degassing under vacuum at $150{ }^{\circ} \mathrm{C}$ for $1.5 \mathrm{~h}$. Once degassed, the lead oleate solution is switched from vacuum to nitrogen positive pressure; the temperature is increased to $250{ }^{\circ} \mathrm{C}$, and $4 \mathrm{~mL}$ of $0.37 \mathrm{M}$ TOP-Te is rapidly injected. The mixture is near $250^{\circ} \mathrm{C}$ for $9 \mathrm{~min}$ and then cooled rapidly to room temperature by immersing the flask in a water bath. The product is precipitated by adding acetone and centrifuging, after which it is redissolved in hexane. This washing procedure is repeated three times. Small bundles of glass fiber are coated and annealed as previously described except that $4 \%$ hydrogen diluted in nitrogen is used during annealing to minimize the chance of explosion.

The low magnification transmission electron microscopy (TEM) image (Figure 1A) shows cubic nanocrystals with an edge length distribution of $12.9 \pm 1.1 \mathrm{~nm}$ (Figure 1A inset). The high-resolution transmission electron microscopy (HRTEM) image (Figure 1B) shows lattice fringes with a spacing of $0.32 \mathrm{~nm}$, which corresponds to the $\{200\}$ planes of PbTe (JCPDS 38-1435). X-ray diffraction (XRD) patterns of as-synthesized nanocrystals and of coated and annealed fibers (Figure 1C) further show that the material is pure phase PbTe. Energy dispersive X-ray spectroscopy (EDX) of as-synthesized nanocrystals (Figure 1D) indicates that the material is slightly tellurium rich. High magnification scanning electron microscopy (SEM) studies of the coated fiber surface elucidate the effect of annealing. Figure $1 \mathrm{E}$ shows that the as-made nanocrystal coating is smooth with $1-2 \mathrm{~nm}$ gaps in between adjacent nanocrystals before annealing. Figure $1 \mathrm{~F}$ shows that, after annealing, the coating is rough with groups of agglomerated nanocrystals, creating a more effective network for charge carrier transport. All subsequently discussed characterization and measurements are performed on annealed fibers.

To investigate the effect of fiber composition on the nanocrystal/glass composite thermal conductivity, we prepare six fiber samples with various nanocrystal coating thicknesses by varying the number of dip coating cycles from 0 to 16 . Samples $1-3$ are dip coated 0 times and, hence, contain $0 \% \mathrm{PbTe}$ by volume. Samples 4, 5, and 6 are dip coated 4, 8, and 16 times and contain $2.9 \pm 1.4 \%, 5.8 \pm 1.6 \%$, and $35.8 \pm 7.6 \%$ PbTe by volume, respectively. For samples $1-5$, a thin layer of gold or platinum is evaporated or sputtered onto the surface of the fiber to make the samples electrically conductive enough for stable $3 \omega$ measurements. Figure 2 shows cross section images of sample 1 and samples 4-6; it illustrates how the increased number of dip coating cycles causes a monotonic increase in the coating thickness and therefore the volume fraction of PbTe.

For $3 \omega$ measurements a single fiber is cut from a coated and annealed bundle and carefully placed on four colinear silver epoxy electrodes, which are subsequently cured at $125{ }^{\circ} \mathrm{C}$ for $15 \mathrm{~min}$ in a nitrogen glovebox. The measurements are performed on a variable temperature stage inside a microprobe station as shown schematically in Figure 3A. While thermal radiation is not eliminated by this experimental setup (vide infra), convection effects are minimized by performing the experiment under vacuum (less than $0.075 \mathrm{mTorr}$ ). An electrically insulating, thermally conducting sapphire substrate is used to ensure that the four electrodes are thermally grounded to the temperature controlled stage. Given the high thermal conductivities of sapphire $(\sim 25-45 \mathrm{~W} / \mathrm{mK})^{24}$ and silver epoxy $(29 \mathrm{~W} / \mathrm{mK})^{25}$ compared with the expected thermal conductivity and small diameter of the coated fibers (0.5-0.93 $\mathrm{W} / \mathrm{mK}, 10-14 \mu \mathrm{m})$, the heat transfer resistance of the fiber greatly exceeds that of the epoxy and sapphire such that the 


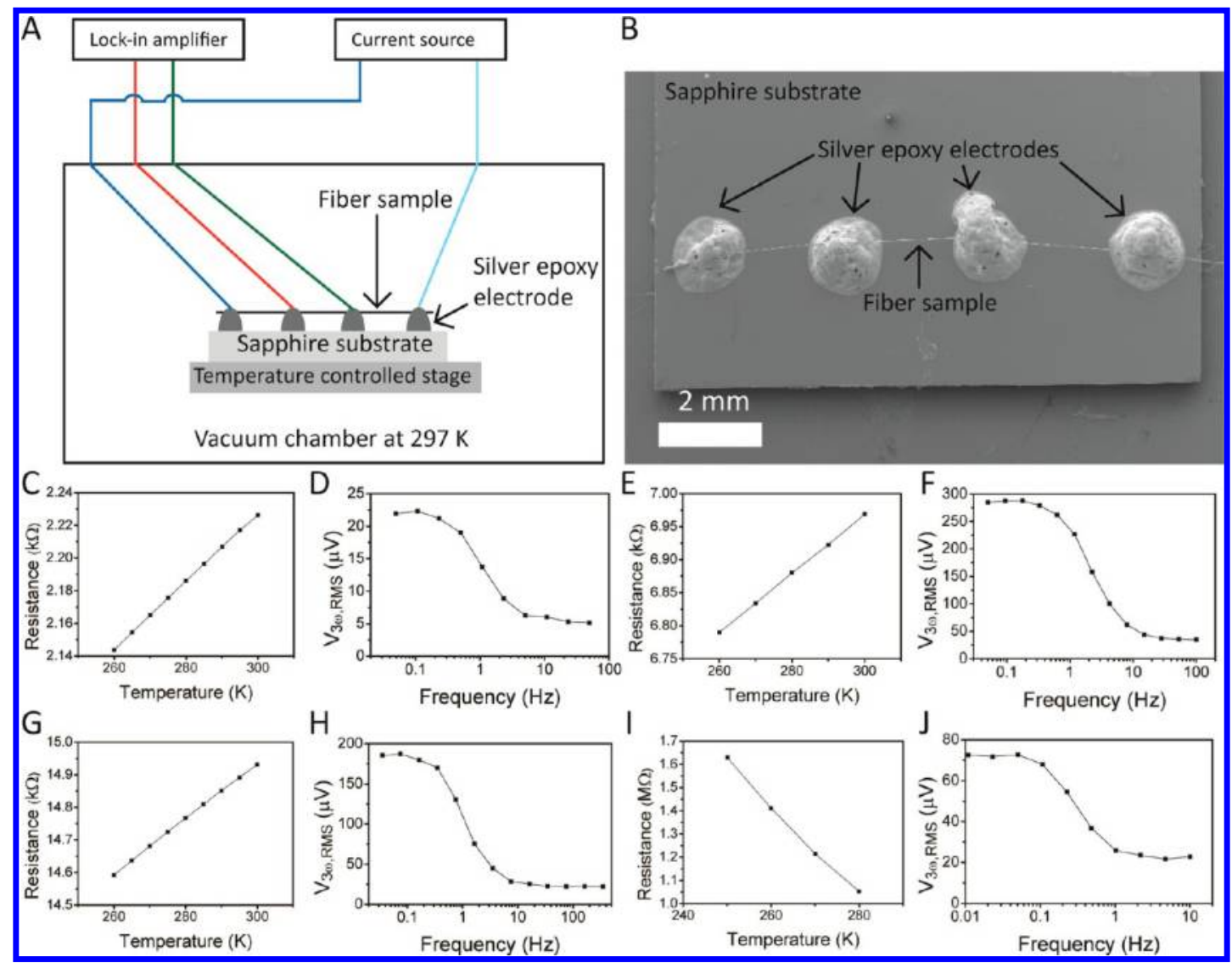

Figure 3. (A) Schematic of $3 \omega$ measurement apparatus. Colored lines represent electrical leads. (B) SEM image of the fiber sample, electrodes, and substrate used for $3 \omega$ measurements. (C-J) Resistance vs stage temperature plots and near room temperature $3 \omega$ voltage vs frequency plots for coated glass fibers. (C, D) Sample 1: 0\% PbTe. (E, F) Sample 4: 2.9\% PbTe. (G, H) Sample 5: 5.8\% PbTe. (I, J) Sample 6: 35.8\% PbTe.

stage-sapphire-epoxy system should effectively be isothermal. A scanning electron micrograph of the fiber, electrodes, and sapphire substrate is shown in Figure 3B.

At each temperature investigated, sinusoidal current is sourced through the outer electrodes, and the root-meansquared (RMS) $1 \omega$ voltage across the inner electrodes is measured using a lock-in amplifier as shown in Figure 3A. The sample resistance was determined by the slope of the $1 \omega$ RMS voltage $\left(V_{1 \omega, \mathrm{RMS}}\right)$ versus the RMS current $\left(I_{\mathrm{RMS}}\right)$. Representative current-voltage data for all samples are given in Figure S1 in the Supporting Information ${ }^{26}$ and show linear relationships in all cases. The variation of resistance with stage temperature for samples of varying PbTe coating thickness is shown in Figure 3C, E, G, and I, from which the value of $R^{\prime}$ can be determined using the derivative of the best linear or polynomial fit.

Next, sinusoidal current in the range of $0.01-400 \mathrm{~Hz}$ is sourced, and the $3 \omega$ voltage is measured by the lock-in amplifier. Prior to all lock-in amplifier measurements, a minimum of 33 periods (inverse of source current frequency) elapse to reach time-invariant ac voltages across the inner electrodes. At each temperature and frequency, 10 ac voltage measurements are taken and averaged to increase measurement accuracy. According to the analytical expression given by $\mathrm{Lu}$ et al., ${ }^{3}$ as frequency decreases, the RMS $3 \omega$ voltage should gradually increase and then asymptotically approach a frequency independent value. Figure $3 \mathrm{D}, \mathrm{F}, \mathrm{H}, \mathrm{J}$ shows the RMS $3 \omega$ voltage as a function of input current frequency for samples with different coating thicknesses. For each sample, the
RMS $3 \omega$ voltage reaches a frequency-independent value at low frequency. The agreement between experiments and theory is further verified by observing the relationship, $V_{3 \omega, \mathrm{RMS}} \sim \mathrm{I}_{\mathrm{RMS}}{ }^{3}$, for several samples as shown in Figure S3 in the Supporting Information. $^{26}$

According to the series solution described by Lu et al., ${ }^{3}$ the thermal conductivity in the low frequency limit is given by eq 1

$$
\kappa=\frac{4 I_{\mathrm{RMS}}^{3} R R^{\prime} L}{\pi^{4} V_{3 \omega, \mathrm{RMS}} S}
$$

where $R^{\prime}$ is the derivative of resistance with respect to temperature, $L$ is the sample length, $S$ is the sample cross section area, and $V_{3 \omega \text {, RMS }}$ is the RMS $3 \omega$ voltage. The effect of time varying $(\mathrm{ac})$ radiation on the self-heated wire $3 \omega$ method is discussed by $\mathrm{Lu}$ et $\mathrm{al}^{3}{ }^{3}$ for experiments in which the environment temperature is equal to the electrode temperature. The ac radiation corrected thermal conductivity at low frequency is given by eq 2

$$
\kappa=\frac{4 I_{\mathrm{RMS}}^{3} R R^{\prime} L}{\pi^{4} V_{3 \omega, \mathrm{RMS}} S}-\frac{16 \varepsilon \sigma T_{0}^{3} L^{2}}{\pi^{2} \mathrm{D}}
$$

where $\varepsilon$ is the sample emissivity, $\sigma$ is the Stefan-Boltzmann constant, $T_{0}$ is the electrode temperature, and $D$ is the sample diameter. The thermal conductivity values calculated by eqs 1 and 2 for sample 6 are shown in Figure 4A using a total emissivity value of 0.736 measured separately by Thermophysical Properties Research Laboratory, Inc. 


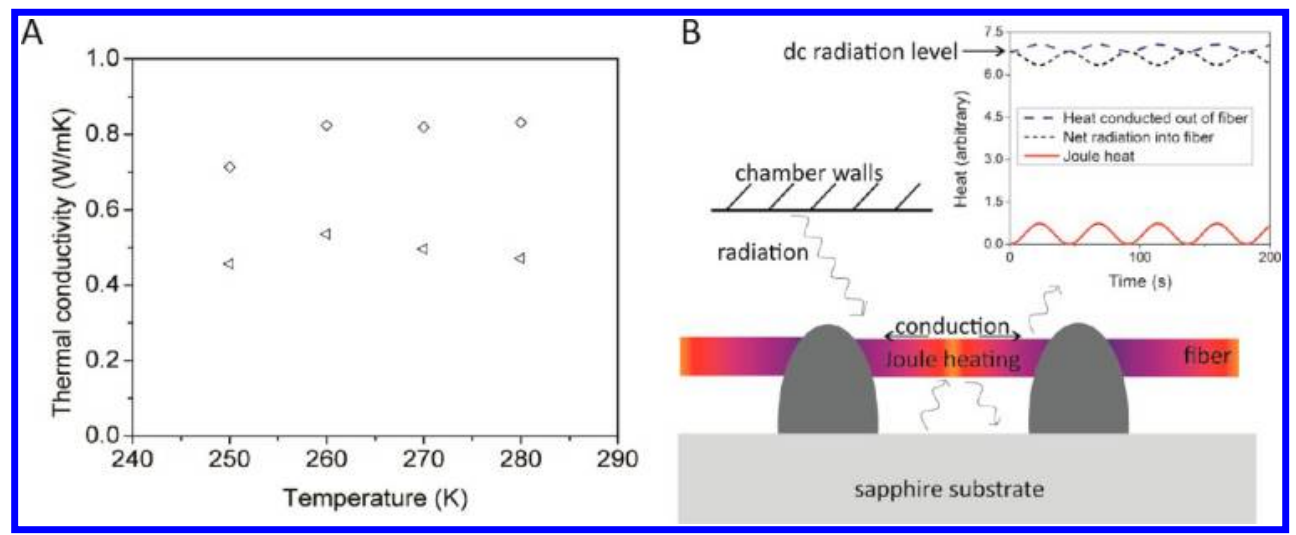

Figure 4. (A) Thermal conductivity of sample 6 as calculated by eqs 1 (diamonds) and 2 (triangles). The dc radiation effect is not accounted for in these results. (B) Schematic of the heat transfer occurring during the $3 \omega$ measurement of the coated fiber. The graph shows how the dc radiation level is nonzero due to the temperature difference between the chamber walls and the fiber.

Equation 2 is valid for experiments performed in a uniformtemperature cryostat. However, in our experiments the temperature controlled stage-sapphire-epoxy system is varied from 250 to $310 \mathrm{~K}$, while the probe station chamber environment remains at $297 \mathrm{~K}$. As approximately half of the fiber surface is exposed to radiation from a room temperature environment, radiation heats/cools the fiber, resulting in a dc temperature rise/fall in the fiber when the stage temperature is below/above $297 \mathrm{~K}$. Figure 4B shows a fiber below room temperature being heated by radiation such that the average temperature is slightly higher than that of the electrodes and sapphire stage. The inset graph shows how the net radiation into the sample includes an ac fluctuation as well as a time invariant (dc) offset that is not considered in eq 2 . The energy balance for this complicated heat transfer problem includes a $T^{4}$ term due to the dc radiation effect. Therefore an analytical derivation was abandoned in favor of a numerical simulation like that performed by Feng et al. ${ }^{14}$

To extract the accurate thermal conductivity while considering the radiation heating, we use the finite difference method to simulate the $3 \omega$ measurement process. Heat conduction is considered as $1 \mathrm{D}$ along the axial direction, and the thermal radiation between the fiber and the surroundings (the bottom half with the substrate and the upper half with the chamber walls, as illustrated in Figure 4B) can be safely simplified as a distributed heat source/sink in the modeling. The above assumptions can be justified by analyzing the Biot number of the fiber in the radial direction, which can be estimated as $B i=h_{\mathrm{r}} r / K$, where $h_{\mathrm{r}}$ is the effective radiative heat transfer coefficient, $r$ is the radius of the fiber $(D / 2 \leq 7 \mu \mathrm{m})$, and $\kappa$ is the thermal conductivity $(\kappa>0.2 \mathrm{~W} / \mathrm{mK}) . h_{\mathrm{r}}$ can be calculated as

$$
h_{\mathrm{r}}=\varepsilon \sigma \frac{\left(T_{\text {surface }}^{4}-T_{\text {surroundings }}^{4}\right)}{\left(T_{\text {surface }}-T_{\text {surroundings }}\right)} \approx 4 \varepsilon \sigma \bar{T}^{3}
$$

where $\bar{T}$ is the average temperature of the surface of the fiber $\left(T_{\text {surface }}\right)$ and the surroundings $\left(T_{\text {surroundings }}\right)$. In our experiment, $\bar{T}$ always satisfies $\bar{T}<310 \mathrm{~K}$, so we calculate $B i \leq 2 \varepsilon \sigma \bar{T}^{3} \mathrm{D} / \kappa \approx$ $1 \times 10^{-4} \ll 0.1$, which means that the $1 \mathrm{D}$ heat conduction model is accurate for this fiber.

The thermal and electrical conduction follow Fourier's law and Ohm's law, respectively. The transient voltage between the two inner electrodes recorded in the simulation is approximately a sine wave with frequency $\omega$, as shown in the inset of
Figure 5A. A Fourier transform of this voltage signal can thus be used to extracted the voltage of the $3 \omega$ signal. As shown in Figure $5 \mathrm{~A}$, the $3 \omega$ signal can be identified clearly in the frequency domain. If the simulated $3 \omega$ voltage agrees with the experimentally measured one, the value of $\kappa$ used in the simulation is taken as the actual $\kappa$ at that temperature. To extract accurate $3 \omega$ voltages, an accurate relation between the resistance and the temperature $(T)$ is needed. Due to the existence of thermal radiation, the experiment only yields $R$ as a function of the stage temperature $\left(T_{s}\right)$ rather than the wire temperature $T$. To extract the $R-T$ relation from the $R-T_{\mathrm{s}}$ relation, we need to derive the average $T$ of the fiber at each $T_{\mathrm{s}}$, which requires the knowledge of the $\kappa-T$ relation. Therefore, the problem needs to be solved in an iterative manner. When the iterative process converges to a final $\kappa-T$ relation, the contribution of the gold or platinum to the thermal conductivity in samples $1-5$ is subtracted using eq $S 1$ in the Supporting Information ${ }^{26}$ and considering the measured resistance and Lorenz numbers for such thin films given in literature. $^{27,28}$

As a verification of our simulation, we compare the simulation results with the analytical solutions ${ }^{3}$ for the onedimensional case without radiation in the low-frequency limit, and good agreement is found, as shown in Figure 5B. Figure 5C shows the thermal radiation effect on the time-averaged temperature profiles at different $T_{\mathrm{s}}$ for sample 6 . The average temperature $T$ deviates approximately $4-8 \mathrm{~K}$ from $T_{\mathrm{s}}$ in certain cases, and this distorts the $R-T$ relation significantly if we neglect such effect, as shown in Figure 5D. The iteration procedure for calculating $\kappa$ shows good convergence, and $\kappa$ is found to be $0.5-0.6 \mathrm{~W} / \mathrm{mK}$ at $250-280 \mathrm{~K}$ (Figure $5 \mathrm{E}$ ). We can also see that $\kappa$ can be overestimated by eq 1 and underestimated by eq 2, primarily caused by the inaccurate temperature dependence of resistivity used due to the deviation of $T$ from substrate temperature $T_{s}$.

Figure 5F shows the thermal conductivity of the $\mathrm{PbTe}$ nanocrystal coated glass fiber as a function of $\mathrm{PbTe}$ volume fraction. The thermal conductivity of bare glass fiber is found to be $0.70 \pm 0.10 \mathrm{~W} / \mathrm{mK}$, which is lower than results for bulk Pyrex glass in literature. ${ }^{29-31}$ We observe that, with the addition of a thin layer of PbTe nanocrystals, the fiber thermal conductivity initially increases to $0.93 \pm 0.14 \mathrm{~W} / \mathrm{mK}$ for the composite with $5.8 \% \mathrm{PbTe}$ but then decreases to $0.55 \pm 0.12$ $\mathrm{W} / \mathrm{mK}$ for the composite with $35.8 \% \mathrm{PbTe}$. This nonmonotonic trend could be explained by the onset of cracking 


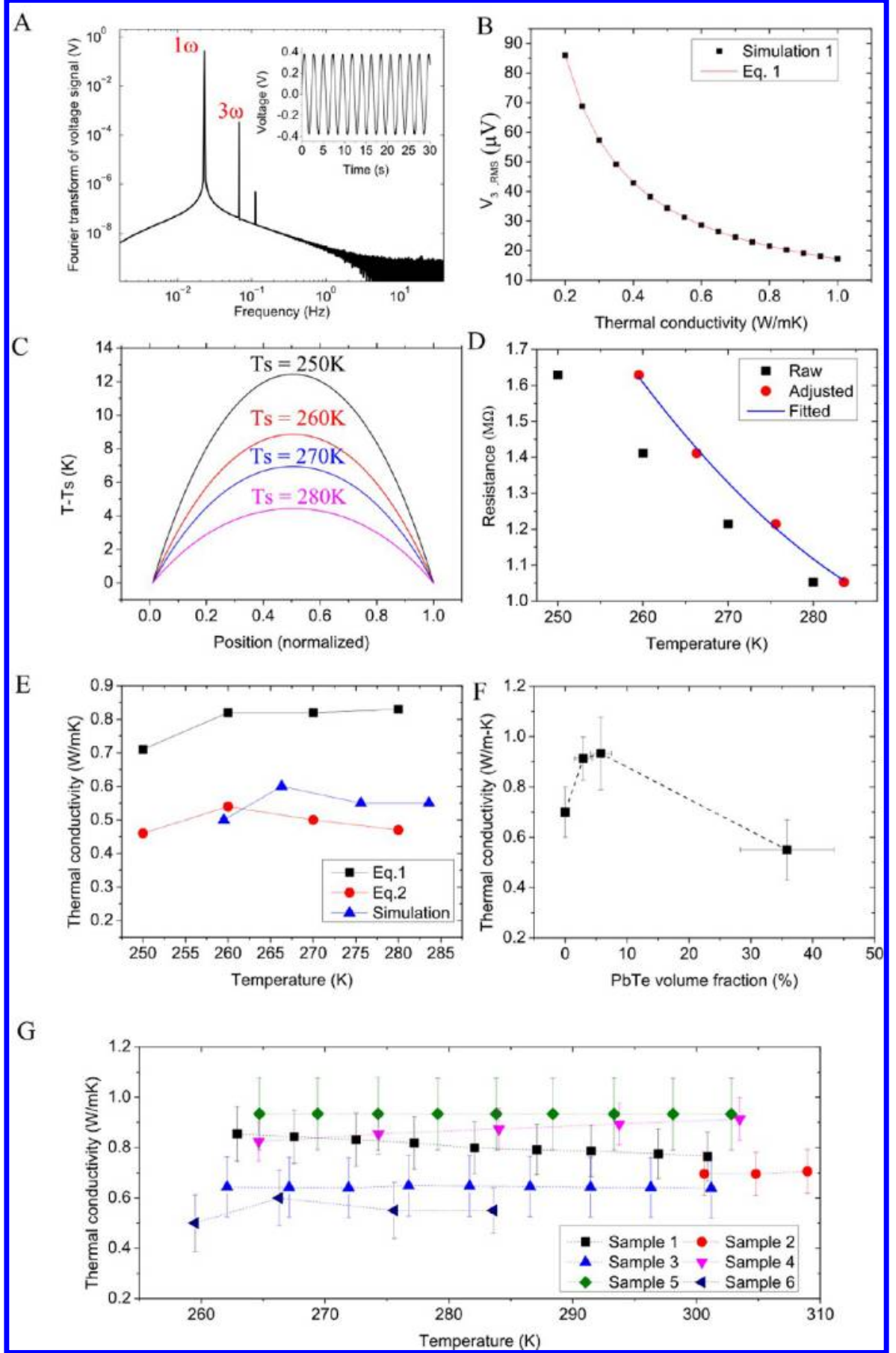

Figure 5. (A) Fourier transformation of the voltage signal (inset) in the simulation. The primary voltage ( $1 \omega)$ and the $3 \omega$ voltage can be extracted as shown. (B) Comparison between the analytical solutions to the $3 \omega$ voltage given in eq 1 and our simulation results in the low-frequency limit when no thermal radiation is included. (C) Time-averaged temperature profiles relative to the substrate temperature $T_{\mathrm{s}}$ between the two inner electrodes when the thermal radiation between the fiber and the surroundings is considered. (D) Temperature-dependent resistance of the fiber before (raw) and after (adjusted) the iteration process and the quadratically fitted adjusted relation. (E) Comparison between the thermal conductivity of sample 6 as a function of temperature calculated by eqs 1 and 2 and by simulation. (F) Thermal conductivity of PbTe coated glass fiber as a function of $\mathrm{PbTe}$ volume fraction around room temperature. $(\mathrm{G})$ Temperature-dependent thermal conductivity of all samples.

in samples with thick nanocrystal coatings. As shown in Figure $\mathrm{S} 11$ in the Supporting Information, ${ }^{26}$ the thin PbTe coatings on samples 4 and 5 are nearly crack-free coatings, while the thick $\mathrm{PbTe}$ coating on sample 6 contains many cracks, which could impede heat transport. In Figure 5G, we plot the temperaturedependent thermal conductivity for six samples fabricated in our experiment. The thermal conductivity of each sample does not change much in the range of temperatures studied in our experiment, but it differs considerably between different samples, due to the difference in $\mathrm{PbTe}$ volume fraction as well as inherent measurement uncertainty.

In conclusion, we have investigated the thermal conductivity of lead telluride nanocrystal coated glass fibers in the axial direction at the single fiber level using the self-heated $3 \omega$ 
technique. The low thermal conductivity of the sample and the $\mathrm{dc}$ radiation from the room temperature microprobe station walls violate assumptions inherent in the analytical expressions derived by $\mathrm{Lu}$ et al. Therefore, a finite difference method has been used to accurately simulate the $3 \omega$ measurements with the radiation effect. By iterating thermal conductivity values in the simulations to obtain agreement with experimental $3 \omega$ voltages, the thermal conductivity was found to be $0.50-0.93 \mathrm{~W} / \mathrm{mK}$ in the temperature range $260-310 \mathrm{~K}$. We also observe that fibers with small volume fractions of $\mathrm{PbTe}$ possess an increase in thermal conductivity compared to bare glass. Meanwhile, cracking in thick nanocrystal coatings result in a decrease in thermal conductivity for fibers with a high volume fraction of PbTe. We expect that our process of including both an experimental approach and numerical modeling could be useful to study the thermal conductivity of other wire-like samples with a low thermal conductivity and high aspect ratio at the single wire level, thus significantly broadening the application of the $3 \omega$ measurement technique.

\section{ASSOCIATED CONTENT}

\section{S Supporting Information}

Additional sample preparation details, current vs voltage data, additional resistance vs temperature and $3 \omega$ voltage vs frequency data, emissivity measurement details and results, and simulation details for all measured samples. This material is available free of charge via the Internet at http://pubs.acs.org.

\section{AUTHOR INFORMATION}

\section{Corresponding Authors}

*Tel.: +1-765-494-5721. E-mail: ruan@purdue.edu. Fax: +1765-494-0539.

*Tel.: +1-765-494-6028. E-mail: yuewu@purdue.edu. Fax: +1765-494-0805.

\section{Author Contributions}

${ }^{\#}$ S.W.F. and Y. Wang contributed equally.

\section{Notes}

The authors declare no competing financial interest.

\section{ACKNOWLEDGMENTS}

Y. Wu thanks the support from NSF/DOE Thermoelectric Partnership (Award no. 1048616) and the support from Air Force Office of Scientific Research (Award no. FA9550-12-10061). Y. Wang and X.R. acknowledge the partial support from National Science Foundation (Award no. CBET-1150948). Y. $\mathrm{Wu}$ and S.W.F. thank the support from the ASEE SFFP.

\section{REFERENCES}

(1) See, K. C.; Feser, J. P.; Chen, C. E.; Majumdar, A.; Urban, J. J.; Segalman, R. A. Nano Lett. 2010, 10 (11), 4664-4667.

(2) Liang, D.; Yang, H.; Finefrock, S. W.; Wu, Y. Nano Lett. 2012, 12 (4), 2140-2145.

(3) Lu, L.; Yi, W.; Zhang, D. L. Rev. Sci. Instrum. 2001, 72 (7), 29963003.

(4) Moon, J.; Weaver, K.; Feng, B.; Chae, H. G.; Kumar, S.; Baek, J.B.; Peterson, G. P. Rev. Sci. Instrum. 2012, 83 (1), 016103-3.

(5) Demko, M. T.; Dai, Z.; Yan, H.; King, W. P.; Cakmak, M.; Abramson, A. R. Rev. Sci. Instrum. 2009, 80 (3), 036103-3.

(6) Hou, J.; Wang, X.; Zhang, L. Appl. Phys. Lett. 2006, 89 (15), 152504-3.

(7) Guo, J.; Wang, X.; Geohegan, D. B.; Eres, G.; Vincent, C. J. Appl. Phys. 2008, 103 (11), 113505-9.
(8) Boukai, A. I.; Bunimovich, Y.; Tahir-Kheli, J.; Yu, J.-K.; Goddard, W. A., III; Heath, J. R. Nature 2008, 451 (7175), 168-171.

(9) Hochbaum, A. I.; Chen, R.; Delgado, R. D.; Liang, W.; Garnett, E. C.; Najarian, M.; Majumdar, A.; Yang, P. Nature 2008, 451 (7175), 163-167.

(10) Li, D.; Wu, Y.; Kim, P.; Shi, L.; Yang, P.; Majumdar, A. Appl. Phys. Lett. 2003, 83 (14), 2934-2936.

(11) Shi, L.; Li, D.; Yu, C.; Jang, W.; Kim, D.; Yao, Z.; Kim, P.; Majumdar, A. J. Heat Transfer 2003, 125 (5), 881-888.

(12) Wang, Z. L.; Tang, D. W.; Zhang, W. G. J. Phys. D: Appl. Phys. 2007, 40 (15), 4686.

(13) Aliev, A. E.; Guthy, C.; Zhang, M.; Fang, S.; Zakhidov, A. A.; Fischer, J. E.; Baughman, R. H. Carbon 2007, 45 (15), 2880-2888.

(14) Feng, B.; Li, Z.; Zhang, X.; Peterson, G. P. J. Vac. Sci. Technol., B 2009, 27 (5), 2280-2285.

(15) Hopkins, P. E.; Phinney, L. M. J. Heat Transfer 2009, 131 (4), 043201-8.

(16) Cahill, D. G.; Katiyar, M.; Abelson, J. R. Phys. Rev. B 1994, 50 (9), 6077-6081.

(17) Liu, W.; Balandin, A. A. Appl. Phys. Lett. 2004, 85 (22), 52305232.

(18) Coates, N. E.; Yee, S. K.; McCulloch, B.; See, K. C.; Majumdar, A.; Segalman, R. A.; Urban, J. J. Adv. Mater. 2013, 25 (11), 16291633.

(19) Hakansson, E.; Kaynak, A.; Lin, T.; Nahavandi, S.; Jones, T.; Hu, E. Synth. Met. 2004, 144 (1), 21-28.

(20) Lin, T.; Wang, L.; Wang, X.; Kaynak, A. Thin Solid Films 2005, $479(1-2), 77-82$

(21) Boakye, E.; Hay, R. S.; Petry, M. D. J. Am. Ceram. Soc. 1999, 82 (9), 2321-2331.

(22) Boakye, E. E.; Hay, R. S.; Petry, M. D.; Parthasarathy, T. A. J. Am. Ceram. Soc. 2004, 87 (10), 1967-1976.

(23) Boakye, E. E.; Hay, R. S.; Mogilevsky, P.; Cinibulk, M. K. J. Am. Ceram. Soc. 2008, 91 (1), 17-25.

(24) Liu, W.; Balandin, A. A. J. Appl. Phys. 2005, 97 (7), 073710-6.

(25) EPO-TEK H20E Technical Data Sheet. https://www. laddresearch.com/index.php/lanotattachments/download/file/id/6/ store/1/, May 2009.

(26) Additional details on sample preparation, emissivity measurement and iterative simulation procedure in computing thermal conductivity from measured data can be found in the Supporting Information.

(27) Lin, H.; Xu, S.; Li, C.; Dong, H.; Wang, X. Nanoscale 2013, 5 (11), 4652-4656.

(28) Zhang, X.; Xie, H.; Fujii, M.; Ago, H.; Takahashi, K.; Ikuta, T.; Abe, H.; Shimizu, T. Appl. Phys. Lett. 2005, 86 (17), 171912-3.

(29) Yang, G.; Migone, A. D.; Johnson, K. W. Phys. Rev. B 1992, 45 (1), 157-160.

(30) Log, T.; Metallinou, M. M. Rev. Sci. Instrum. 1992, 63 (8), 3966-3971.

(31) Miller, M. S.; Kotlar, A. J. Rev. Sci. Instrum. 1993, 64 (10), $2954-2960$. 